\title{
On Backoff in Fading Wireless Channels ${ }^{\star}$
}

\author{
SeonYeong Han and Nael B. Abu-Ghazaleh \\ Computer Science Dept. \\ State University of New York at Binghamton \\ and \\ School of Computer Science \\ Carnegie Mellon University - Qatar \\ \{shan6@,nael@cs.\}binghamton.edu
}

\begin{abstract}
We consider the impact of transmission errors on the backoff algorithm behavior in the IEEE 802.11 protocol. Specifically, since the backoff algorithm assumes that all packet losses are due to collisions, it unnecessarily backs off when a packet is lost due to a transmission error. Two performance problems arise as a result: (1) low throughput, due to unnecessary loss of transmission time; and (2) unfairness when two competing links have different transmission error rates. In this paper, we characterize this problem and propose three solutions to it. The solutions aim to provide discrimination between transmission errors and collisions such that the sender can back off appropriately. The first algorithm relies on receiver discrimination and feedback; the receiving radio can in many instances differentiate between collisions and transmission errors. The second algorithm estimates the clear channel quality, and backs off if the observed quality deviates from the clear channel quality (indicating collisions). The third algorithm develops the probability of collision as a function of the number of observed idle slots during contention, and uses this probability to control the backoff algorithm. We show via simulation that the techniques significantly improve both performance and fairness of IEEE 802.11 in the presence of transmission errors.
\end{abstract}

\section{Introduction}

The IEEE 802.11 MAC protocol [8] is the de facto standard for wireless LANs, including ad hoc and mesh networks. It is a contention based protocol that uses Carrier Sense Multiple Access with Collision Avoidance (CSMA/CA) to reduce the probability of collisions. In contention protocols, collisions cannot be eliminated; this is especially true for wireless networks due to the well-known hidden terminal problem $[12,6]$. Thus, an important component of contention MAC protocols is the backoff mechanism which is used to regulate the offered load to the shared channel in the presence of contention. Specifically, when a collision occurs Binary Exponential Backoff (BEB), is invoked, typically doubling

* This work is partially supported by NSF grant CNS-0454298 
the size of the backoff window. BEB is used in other contention protocols such as Ethernet.

IEEE 802.11 interprets all packet losses as collisions and invokes the BEB algorithm. However, in the presence of wireless transmission errors, the BEB protocol is invoked unnecessarily (since a transmission error is not an indication of contention), leading to the following two performance problems: (1) Inefficient use of the available bandwidth: this is a consequence of unnecessarily increasing the backoff window. This problem is exacerbated when one considers that contention occurs in IEEE 802.11 using the lowest transmission rate, to allow fair contention among connections with heterogeneous rates. As a result, at higher rates, data packet transmission time becomes shorter, but the backoff period stays the same as the lowest rate;

(2)Unfairness: when two links with different loss rates are in interference range of each other, unfairness arises. The weaker link backs off more frequently due to transmission errors, creating unfair competition for the medium and longterm unfairness. We characterize the impact of fading on IEEE 802.11 performance under different scenarios in Section 2.

This paper contributes three solutions for remedying these problems. A successful solution should discriminate between losses due to collisions and those due to transmission errors. This discrimination does not necessarily have to be at the granularity of the individual transmission; rather, the technique should provide insight into the probability of a loss in the aggregate being due to collisions. We investigate the following three solutions, which are presented in more detail in Section 3.

1. Receiver based discrimination: in this solution, the receiver uses any information available to it to determine the cause of the packet loss. Increasingly, the physical layer at the receiver is able to provide information about the transmission that is helpful in speculating on the reason for the loss. The speculation results are fed back to the sender on subsequent acknowledgments, allowing it to adjust its backoff window proportionately. This approach relies critically on the discrimination mechanism at the receiver and the information available from the wireless card.

2. Link Quality Estimation: in this solution, the sender maintains a running estimate of the clear channel link quality (the expected loss rate in the absence of contention from other sources). The backoff window is then increased in proportion to the loss rate being observed vs. that expected by the link quality. However, estimating the clear channel link quality while the network is active is difficult. We take an approach in which we use the minimum loss rate period over a period of time as an estimate of the clear channel link quality.

3. Idle Slot Collision Probability Estimation: Heusse et al. [7] observed that the number of idle slots in a contention period is indicative of the amount of local contention for the use of the channel. We adapt the approach to estimate the probability of collision as a function of the number of idle slots observed. With an estimate of the collision probability, we can estimate the 
number of extra losses that are due to transmission errors and adjust the backoff accordingly.

Section 4 presents a simulation-based evaluation of the proposed approaches. The experiments show that all three approaches are able to address the problem, coming close to the performance of a perfect predictor. Section 5 overviews related work. Finally, Section 6 presents some concluding remarks.

\section{Impact of Fading on Binary Exponential Backoff}

The backoff mechanism regulates the offered load to the shared medium. Backoff algorithms maintain a contention window value in units of fixed-size slots, to determine how long to wait before transmission. In IEEE 802.11, there is a minimum contention window $C W_{\min }$ that is used after a successful transmission. $C W$ is doubled whenever a packet loss occurs until it reaches $C W_{\max }$. After every transmission a node picks a number of slots uniformly distributed in the range $[0, C W]$ as its backoff window.

The underlying assumption in these backoff algorithms is that all packet losses are due to collisions. This assumption holds true in wired shared media where transmission errors are exceptionally rare, but not in wireless environments where they are common. When transmission losses occur, backoff is invoked unnecessarily, leading to significant inefficiency and giving rise to longterm unfairness among links with different qualities.

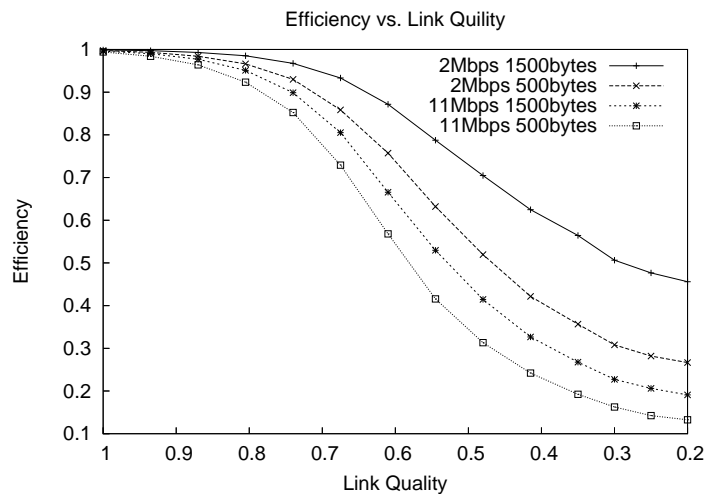

Fig. 1. Efficiency vs. Link Quality

We define efficiency to be the ratio of the observed throughput to the throughput of an ideal backoff algorithm that backs off when collisions occur, but not when transmission errors occur. Figure 1 shows the efficiency on a single hop 
link as a function of the link quality (the probability of successful transmission) for two different transmission rates and packet sizes. Clearly, there is a large drop in throughput as the link quality drops beyond the loss that results from the loss of the packets to transmission errors. Whenever a transmission error occurs, the contention window is doubled unnecessarily. The problem is worse when the packet size is small since the yield from each contention period drops. Further, since contention is carried out at the lowest transmission rate (for compatibility and fairness among transmitters with different rates), the problem also becomes worse when the transmission rate increases. Because the probability of successive transmission errors increases as the link quality degrades (leading to exponential backoff), the efficiency degradation is not linear.

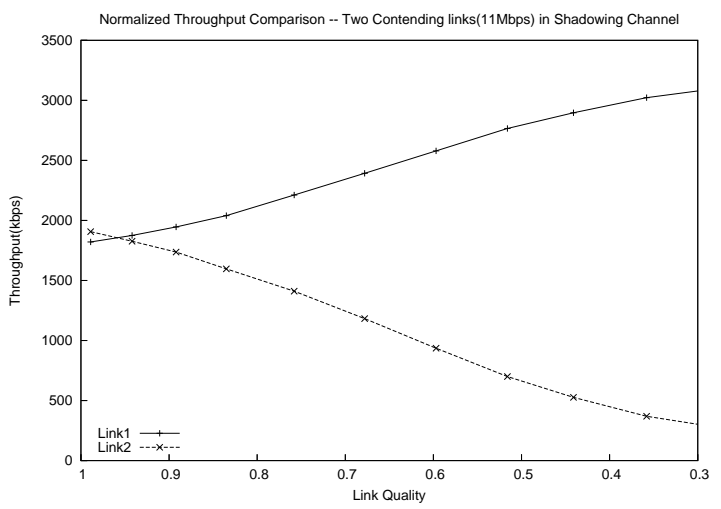

Fig. 2. Unfairness Problem

Unnecessary backoff in response to transmission errors can also give rise to unfairness. When multiple links compete, if the backoff algorithm is not biased towards either, then long term fairness will be achieved. However, in the presence of transmission errors, two links with different loss rates experience different average backoff values. This causes unfair competition in accessing the medium and long term unfairness results. Figure 2 demonstrates this effect between two competing single hop flows. The link quality for the first flow is fixed at 0.95 , while the quality of the second link is varied. The figure plots the normalized throughput (throughput divided by link quality); the normalization is done to remove the effect of the lost packets and provides an estimate of the actual transmissions that each flow receives. Clearly, as the link quality disparity increases, the higher quality links starts dominating the available bandwidth. Discriminating between transmission errors and collisions can mitigate this problem because the weaker quality link is not penalized by the backoff algorithm for transmission errors (which are not indicative of collisions). 


\section{Proposed Solutions}

In this section, we discuss three approaches for informed back off for CSMA based wireless networks. The goal of our solutions is discriminate between transmission losses and collisions so that the back off algorithm increases back off only when collisions occur. An important observation is that this back off does not have to be at the granularity of the individual packet. Instead, an estimate of the percentage of losses due to collisions is sufficient to guide the behavior of the back off algorithm.

Overall, discriminating between transmission errors and collisions is challenging as this information is rarely explicitly and directly discernible for a given transmission. However, often the combined views at the sender and receiver can be used to intelligently and speculatively determine the causes behind packet losses. From the sender's perspective, little information is available about a given packet transmission without receiver feedback. On the other hand, at the receiver, a given lost transmission may be undetected (e.g., due to a deep signal fade or high interference or noise) or partially detected (a part of the packet is corrupted). Both the sender and receiver may collect information about the channel via carrier sense when they are not transmitting to each other; however, the state of the channel at the receiver is more important than the state of the channel at the sender. In the remainder of this section, we propose three solutions to this problem.

\subsection{Receiver based discrimination (RBD)}

Receiver-based discrimination uses the information available at the receiver to identify the cause of a packet loss. At the physical layer, detailed information is available during the packet reception that can allow effective speculation on the reasons behind the packet loss (e.g., [13]). However, commercial wireless cards differ significantly in the information they expose to upper layers. Furthermore, some events are more difficult to detect than others (e.g., a collision or fade during the PLCP header causes complete loss of the packet and no information is available). Therefore, the available information, and the success rate for the speculation, varies significantly with the underlying hardware and drivers. It is possible to explore different alternative algorithms for discrimination based on the information available to the receiver.

Discrimination Mechanism: As an example for this approach, we use a mechanism suggested by Burns et al. for collision detection [4]. For this approach to be effective, the hardware of the receiver should be able to detect the existence of a new packet even though it is currently receiving another packet (indicating a collision). If the receiver is unable to detect the new packet header, it cannot decide if the loss is due to a collision or error. Other approaches for discrimination are possible (e.g., based on the observed RSSI). Once the receiver detects a collision, the speculation results are returned to the sender so that it can adjust its 
back off behavior. We feedback this information opportunistically by including it on subsequent ACKs.

Modified Backoff Algorithm: Once the collision information is received at the sender, the following approach is used to increase the contention window. The conditional collision probability (CCP) that a lost packet is lost due to a collision, rather than a transmission error, is estimated as follows. The receiver feeds back on the ACK $N_{e c o l}$ - the number of packets estimated to have been lost to collisions for a predetermined observation window (in time or number of packets). The estimated CCP is $\frac{N_{\text {ecol }}}{N_{\text {lost }}}$, where $N_{\text {lost }}$ is the total number of lost packets (to errors or collisions) in the same window. Since $N_{\text {lost }}=N_{\text {transmit }}-N_{\text {success }}$, CCP is $\frac{N_{\text {ecol }}}{N_{\text {transmit }}-N_{\text {success }}}$. When a transmission is lost, we back off with probability CCP.

Note that RDB generally underestimates CCP because it can fail to detect some collisions. Moreover, our implementation does not account for ACK losses (which in effect considers all of them to be non-collision losses). However, ACK losses due to collisions are relatively rare because of the small size of the ACK packet. Furthermore, nothing prevents ACK packet collision detection using the same approach.

\subsection{Link Quality Estimation (LQE)}

In this approach, we first estimate the clear channel link quality (CCLQ) which represents the loss rate on the channel in the absence of collisions. Once that is estimated, the expected probability of loss for each transmission can be developed. Over a certain window, again measured in terms of time or number of transmissions, we expect a number of transmission errors based on the number of attempted transmissions and the estimated link quality. Losses exceeding this number can be attributed to collisions and the back off window adjusted accordingly. Note that the approach can be made robust for different packet sizes and/or different transmission rates (e.g., by estimating the bit error rate instead of the packet loss rate). Different flavors of LQE may be developed based on the approach for estimating CCLQ, and how the contention window is adjusted.

Estimating Link Quality: CCLQ may be estimated off-line for static mesh networks by running clear channel measurements while the network is idle. However, this approach is not suitable for dynamic environments and does not adapt to the time-varying nature of link quality. The challenge in dynamically estimating CCLQ is that the channel is not idle while the network is active. Thus, simply tracking the percentage of packets received correctly counts both the losses due to transmission errors and collisions, under-estimating the link quality. We use the highest observed link quality value over a fixed number of measurement windows as the CCLQ. Our intuition is that this high link quality occurs due to a window with few or no collisions. However, the estimate remains approximate: if no period is free of collisions, then the quality is under estimated. Thus, the estimate of CCLQ is heuristic; the heuristic may be improved based on empirical evaluation. Further, LQE expects that the link remains stable over multiple 
windows and is therefore slow in tracking a dynamically changing window (e.g., due to mobility).

Modified Backoff Algorithm: In a given window the probability of loss is computed as the ratio of lost packets to total packets $P_{\text {loss }}$. If $P_{\text {loss }} \leq$ cclq, we update CCLQ to be equal to $P_{\text {loss }}$. However, if $P_{\text {loss }}>c c l q$, we have some collisions. To compute the conditional collision probability, note that

$$
\begin{array}{r}
P_{\text {loss }}=c c l q+P_{c}-c c l q * P_{c} \\
P_{c}=\frac{P_{l o s s}-c c l q}{1-c c l q}
\end{array}
$$

where $P_{c}$ is the probability of collision. The conditional collision probability (CCP) is then $\frac{P_{c}}{P_{\text {loss }}}$; when a transmission is lost, we back off with probability $\mathrm{CCP}$.

\subsection{Idle Slot Collision Probability Estimation (ISCPE)}

Another approach to estimating the conditional collision probability (CCP) relies on the following observation due to Heusse et al [7]. Specifically, they observe that the degree of contention, and hence the probability of collisions, is a function of the number of idle slots after every successful transmission. They use this observation to derive an optimized back off algorithm called IdleSense. IdleSense significantly outperforms Binary Exponential Backoff, but is not compatible with it. Furthermore, IdleSense does not consider transmission losses.

Observing Idle Probability: Because back off algorithm pause the decrement of the back off counter whenever a busy channel is sensed, the length of a run of continuous idle slots is a good indicator of contention level around a receiver. Let the probability that each slot is assigned to some node be $p$, and $q=1-p$. Then, the probability that the $k$ 'th trial is the first success is

$$
\operatorname{Pr}(X=k)=q^{k-1} p
$$

for $k=1,2,3, \ldots$

The random variable $X$ that indicates the number of trials before the first successful slot is geometrically distributed with expected value $E(X)=\frac{1}{p}$. The average observed length of a run of continuous idle slots, $L+1$, is also $E(X)$. Then, $p=1 /(L+1)$ and $q=L /(L+1)$. Because $q$ is the idle probability of a slot,

$$
q=\left(1-P_{e}\right)^{N}
$$

where $N$ represents the number of contending nodes, and $P_{e}$ is the attempt probability per slot. From Eq. $2, P_{e}=1-q^{\frac{1}{N}}$. The idle probability, $P_{i}$, is then $\left(1-P_{e}\right)^{N}=q$.

Because each node senses the idle channel first and then takes the following slot to transmit if its backoff is zero, the minimum $L$ is 1 . Thus, $L=1$ means that the obtained idle probability is overestimated; this is a limitation of idle slot based solution. 
Estimating Collision Probability: According to [7], the successful transmission rate can be estimated as

$$
P_{t}=N \cdot P_{e}\left(1-P_{e}\right)^{N-1}
$$

A slot where a collision occurs is one where more than one transmission occurs. More formally,

$$
P_{c}=1-P_{t}-P_{i}
$$

We can express $P_{c}$ using $P_{i}$ as

$$
P_{c}=1-N\left(1-P_{i}^{\frac{1}{N}}\right) P_{i}^{\frac{N-1}{N}}-P_{i}
$$

$N$ can be found by sensing the channel and $P_{i}$ can be found by observing the number of idle slot after every transmission. Thus, from the computed $P_{c}$ we can estimate CCP, and adjust the back off with that probability on every lost packet.

Estimating the Number of Neighbors: Though ISCPE mostly relies on local information, channel observation is still necessary to determine the number of neighbors, which is needed to calculate the transmission probability and collision probability from the idle probability. In a mobile environment, the observed number of neighbors may be either larger or smaller than the exact number because of tracking lag. The effect of inaccuracy in estimating the number of neighbors will be evaluated in the following section.

\section{Performance Evaluation}

In this section we evaluate the proposed solutions using simulations. For all simulations, we use the NS-2 network simulator [9]. Unless otherwise indicated, we use the log-normal model to simulate a fading channel. We also investigated generating fading losses by training hidden Markov models trained with collected wireless traces, but no appreciable changes in the general trends were observed; therefore we elected to demonstrate the solutions using the more controllable log-normal model.

The first study revisits the case of a single hop flow with varying link quality. Figure 3(a) shows that all three solutions successfully address the backoff problem in the 1-hop case. Since there are no collisions, RBD achieves ideal performance since it detects no collisions and assumes that all losses are due to transmission errors (which is the case). Also, ISCPE will detect an always idle link, correctly predicting that there are no collisions. LQE does not achieve ideal behavior because it incorrectly guesses that a collision occurs whenever the number of losses in a window is above that in the lowest detected window. In other words, since errors do not occur at a constant rate, LQE mispredicts collisions, and therefore its performance is slightly worse than the other schemes.

In the second experiment, we compare the fairness achieved by two contending links using the different backoff algorithms. The quality of one link is fixed 


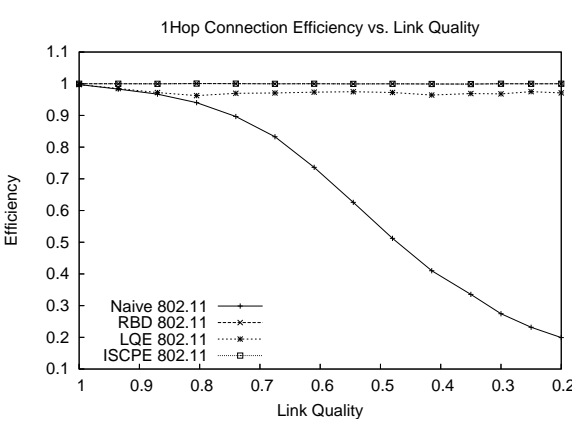

(a) Efficiency vs. Link Quality

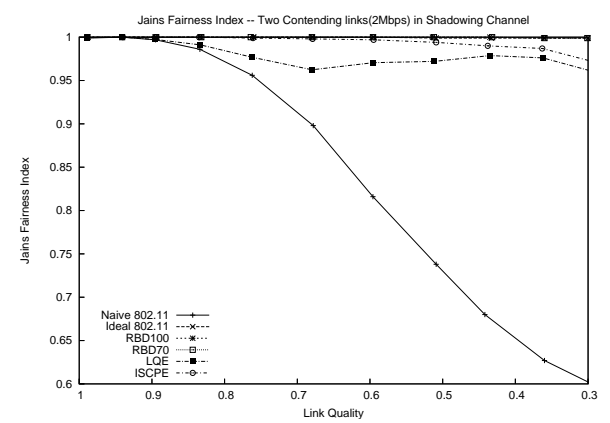

(b) Fairness Comparison in Two Contending Links (11Mbps)

Fig. 3. Effect of the Solutions

at 0.95 , while the quality of the other is varied (x-axis). Figure $3(\mathrm{~b})$ shows the fairness improvement of the three solutions. ISCPE and RBD achieve ideal fairness (Jain's index of 1). RBD calculates the CCD by the periodic observation of the number of transmitted, successfully received, and collided packets. The level of accuracy of collision detection affects fairness,especially with low link quality. If only $70 \%$ of collisions are detected by the receiver,the CCD is underestimated, leading to a smaller contention window than ideal. Surprisingly, a lower accuracy of the RBD does not appreciably harm fairness. Moreover, LQE shows sensitivity to the asymmetric measurement error as link quality decreases. This is because LQE is a solution based on estimated link quality, which is inaccurate if collision loss events occur frequently. However, as can be seen in the figure,fairness improves significantly in all approaches.

We considered a WLAN scenario where five senders and five receivers are in range of each other. In this scenario, neither hidden terminals nor exposed terminals exist; however, collisions due to concurrent transmissions (two or more nodes attempting to transmit in the same slot) can occur [1]; persistent or transient unfairness due to hidden terminals cannot be solved using the schemes in this paper, which focus only on avoiding backoff when transmission losses occur. By fixing the number of transmitters and the transmission rate, the probability of collisions is fixed [1]. In a homogeneous scenario, all five links have the same link quality. In a heterogeneous scenario, link qualities are uniformly distributed in the range of $\left[\operatorname{Min}\left(p_{j}\right), \operatorname{Max}\left(p_{j}\right)\right]$. Each point represents an average of twenty simulation runs to tightly bound the confidence intervals.

Figure 4(a) shows Jain's fairness index for the homogeneous scenario; unfairness is not present because all links have an equal opportunity to access a wireless channel and no persistent asymmetry exists. Jain's fairness index in a heterogeneous scenario is shown in Figure 4(b), where the x-axis represents the lower 


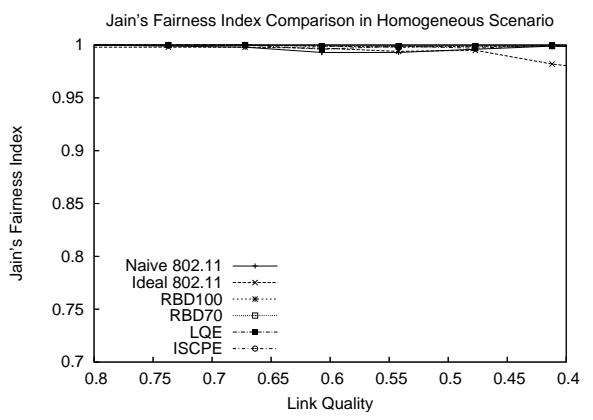

(a) Homogeneous Scenario

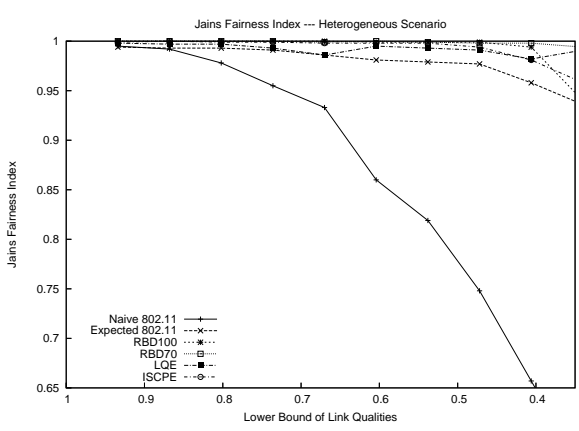

(b) Heterogeneous Scenario

Fig. 4. Jain's Fairness Index

bound of link quality and the y-axis represents the fairness index. In this case, all three proposed solutions dramatically improve unfairness.

Achieving fairness may result in degradation of overall throughput. The primary reason behind this degradation is that by giving more chances to the weaker links to transmit, we end up losing more of the transmitted packets due to errors, harming overall throughput. Thus, aggregate throughput is reduced even though weak links achieve improved throughput.

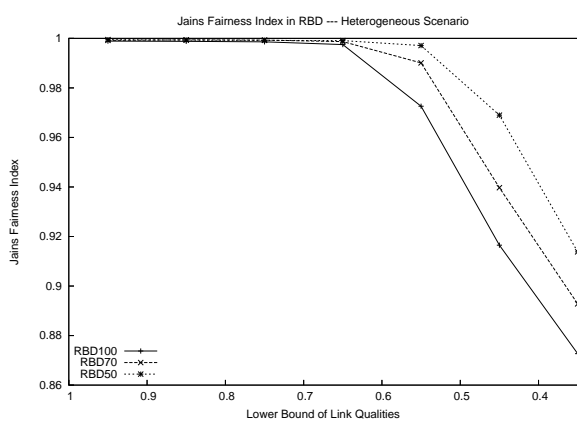

(a) Jain's Fairness Comparison

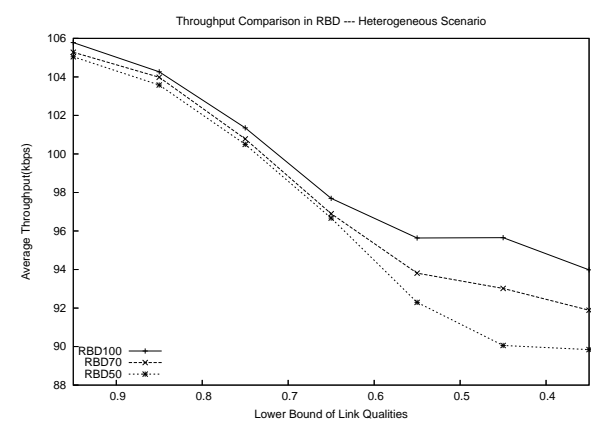

(b) Throughput Comparison

Fig. 5. Comparison in Several Accuracy Level of RBD

Figure 5(a) and 5(b) analyze the impact of discrimination accuracy on the performance of RBD in a heterogeneous scenario. RBDxx indicates that only $\mathrm{xx} \%$ of collisions are detected in the receiver. For the case of RBD50, where only half of the collisions are detected, the fairness increases due to the small 
contention window but average throughput decrease due to collision losses. In fact, our simulation result showed that the number of collisions for RBD70 and RBD50 increase by $3.7 \%$ and $5 \%$ respectively compared to that for RBD100.

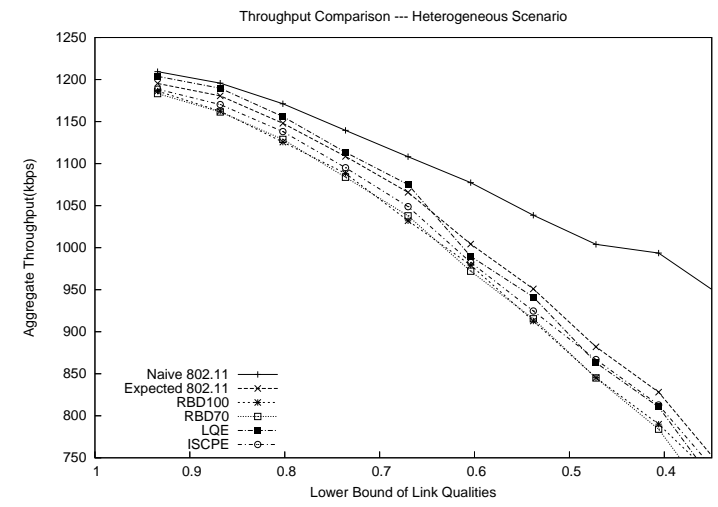

Fig. 6. Throughput Comparison of Solutions

Figure 6 shows aggregate throughput of all proposed solutions. Because the strong links cannot dominate the channel in the solutions, the aggregate throughput of the proposed solutions is lower than that of the naive 802.11.

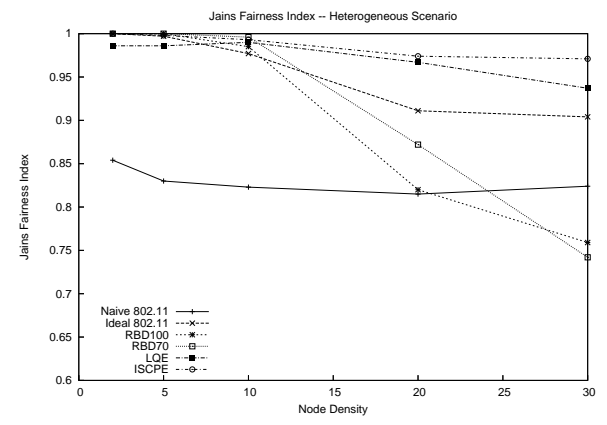

(a) Unfairness Result

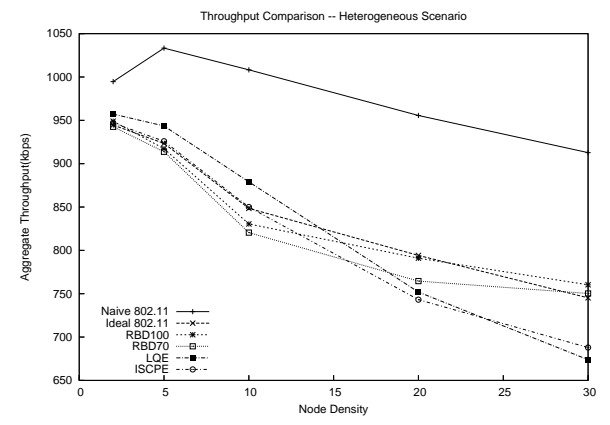

(b) Throughput Result

Fig. 7. Unfairness And Throughput Comparison As The Node Density Increases

Figure 7(a) and 7(b) show the fairness index and throughput as the node density is increased when the lower bound of link qualities is fixed to 0.6. By increasing node density, we increase the collision probability. Naive 802.11 shows 
pretty stable unfairness through the various node density, though the aggregate throughput decreases. This is because the collision losses in a high density scenario happen fairly to the senders, while fading losses happen unfairly. However, the high collision loss due to high node density affects the performance of each solution. RBD needs to refer the returned feedback to estimate collision rate. As the node density increase, the feedback is more likely to be collided with, which results in incomplete feedback. LQE and ISCPE show stable improvement in fairness, because they do not depend on feedback.
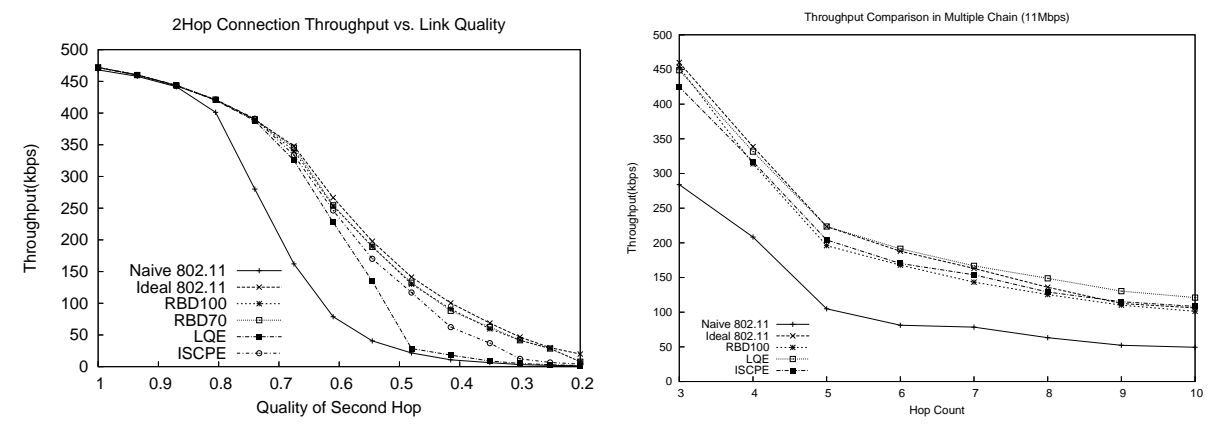

(a) Throughput in a Two-hop Connection (b) Throughput in a Multiple Chain Connection

Fig. 8. Throughput Improvement in a Multi-hop Connection(11Mbps)

Figure 8(a) presents a 2-hop connection throughput improvement for a 500 bytes packet size at $11 \mathrm{Mbps}$, where the first hop has a $95 \%$ link quality and the second hop has various link qualities which are represented on the x-axis. A problem with unfairness in chains where a stronger link is upstream of a weaker link is that the stronger link wins more often, creating a mismatch between input and output at intermediate nodes and consequently packet drops. Increasing fairness, significantly improve performance by eliminating this effect.

The 2-hop scenario is extended to multiple chain connection as shown in Figure 8(b). Link qualities for each hop were assigned randomly. Each point represents an average of twenty simulation runs. All proposed solutions show improved throughput over any hop counts due to increasing fairness among hops, which provides further support for using mechanisms that intelligently back off in the presence of transmission losses. 


\section{Related Work}

Chua and Lye discuss the need to distinguish collisions from errors in timevarying channels [5]. They observe that backoff algorithms should be modified to consider packets that fail due to channel errors, but offer no solution to the problem.

Nadeem et al [11] modify Bianchi's model to study noisy environments. They proposed $\operatorname{smart}_{B E B}$, which adjusts the contention window in increments based on the loss probability $p$. The proposed solution needs time to achieve optimal value. Furthermore, it represents a completely different backoff algorithm that does not inter-operate with the standard IEEE 802.11. The IdleSense algorithm [7] is a similar algorithm to that proposed by Nadeem et al; it also is not inter-operable with IEEE 802.11.

Discrimination of wireless errors from congestion errors has received significant attention with respect to TCP. Since TCP uses packet loss events as an indicator of congestion, it also suffers from undetected wireless losses. Specifically, when a transmission error cause s apacket to be lost, TCP incorrectly activates its congestion control mechanism, resulting in poor performance. Much of the literature proposes end-to-end solutions that analyze the Round Trip Time of received packets $[2,10]$ or use excplicit congestion notification to distinguish the cause of loss [3]. End-to-end solutions do not help to solve the unfairness problem in the MAC layer because they do not influence the backoff algorithm.

\section{Conclusion}

In this paper, we studied the impact of transmission losses on the backoff mechanism of IEEE 802.11. Specifically, the backoff algorithm treats all losses as collision losses, leading to unecessary backoff. The problem leads to two negative side effects: loss of channel time and unfairness. We proposed three different solutions to the problem that attempt to discriminate between transmission errors and collisions then backoff only when a collision occurs. Specifically, Receiver Based Discrimination uses available information at the receiver to determine if a packet loss was due to collisions or errors, and feeds this information back to the sender. Link Quality Estimation estimates the clear channel link quality and backs off whenever the number of losses in a window exceeds the number expected by the link quality (the deviation indicating potential collisions). Finally, we proposed an Idle Slot Collision Probability Estimation mechanism that uses recent results that show that the number of observed idle slots can be used to estimate collision probability and thus to guide backoff behavior. Simulation that the proposed approaches significantly improve the problem and increase throughput and fairness overall. 


\section{References}

1. Bianchi, G. Performance analysis of the ieee 802.11 distributed coordinationfunction. IEEE Journal on Selected Areas in Communications (2000).

2. Biaz, S., AND VAidya, N. H. Discriminating congestion losses from wireless losses using inter-arrival times at the receiver. In ASSET '99: Proceedings of the 1999 IEEE Symposium on Application - Specific Systems and Software Engineering and Technology (Washington, DC, USA, 1999), IEEE Computer Society, p. 10.

3. Biaz, S., And VAidya, N. H. "de-randomizing" congestion losses to improve tcp performance over wired-wireless networks. IEEE/ACM Trans. Netw. 13, 3 (2005), 596-608.

4. Burns, L., Podell, A., Fisher, D., and Ramachandran, R. Radio based collision detection for wireless communication system. US Patent Issued on August 12, 1997.

5. ChuA, K.C.; Lye, K. Backoff considerations in csma/cd lan with single timevarying channel. In Electronics Letters, Volume 27, Issue 9, (25 Apr 1991), pp. 747 $-748$.

6. Garetto, M., Shi, J., And Knightly, E. W. Modeling media access in embedded two-flow topologies of multi-hop wireless networks. In MobiCom '05 (New York, NY, USA, 2005), ACM Press, pp. 200-214.

7. Heusse, M., Rousseau, F., Guillier, R., And Duda, A. Idle sense: an optimal access method for high throughput and fairness in rate diverse wireless lans. SIGCOMM Comput. Commun. Rev. 35, 4 (2005), 121-132.

8. IEEE 802.11 - The WORKING Group FOR Wireless LANs. Ieee 802.11 standard, 1999 edition, 1999. Including IEEE 802.11a and IEEE 802.11b Extension. Available for download from http://www. ieee802.org/11/index.html.

9. Information Sciences Institute. NS-2 network simulator. Software Package, 2005. http://www.isi.edu/nsnam/ns/.

10. Li, Y., Su, F., FAn, Y., AND Xu, H. End-to-end differentiation of congestion and wireless losses using a fuzzy arithmetic based on relative entropy. In International Conference on Systems and Networks Communication (ICSNC'06) (2006), p. 15.

11. Nadeem, T., AND Agrawala, A. Ieee 802.11 def enhancements for noisy environments. In International Symposium on Personal, Indoor and Mobile Radio Communications (PIMRC) (2004).

12. Tobagi, F., And Klienrock, L. Packet switching in radio channels: Part II: The hidden terminal problem in carrier sense multiple access and the busy tone solution. IEEE Transactions on Communication (1975), 1417-1433.

13. Woo, G., Kheradpour, P., Shen, D., and Katabi, D. Beyond the bits: Cooperative packet recovery using physical layer information. In Proc. ACM International Conference on Mobile Computing and Networking (Mobicom) (2007). 